\title{
Continuous anneal method for characterizing the thermal stability of ultraviolet Bragg gratings
}

Rathje, Jacob; Kristensen, Martin; Pedersen, Jens Engholm

Published in:

Journal of Applied Physics

Link to article, DOI:

10.1063/1.373775

Publication date:

2000

Document Version

Publisher's PDF, also known as Version of record

Link back to DTU Orbit

Citation (APA):

Rathje, J., Kristensen, M., \& Pedersen, J. E. (2000). Continuous anneal method for characterizing the thermal stability of ultraviolet Bragg gratings. Journal of Applied Physics, 88(2), 1050-1055.

https://doi.org/10.1063/1.373775

\section{General rights}

Copyright and moral rights for the publications made accessible in the public portal are retained by the authors and/or other copyright owners and it is a condition of accessing publications that users recognise and abide by the legal requirements associated with these rights.

- Users may download and print one copy of any publication from the public portal for the purpose of private study or research.

- You may not further distribute the material or use it for any profit-making activity or commercial gain

- You may freely distribute the URL identifying the publication in the public portal 


\title{
Continuous anneal method for characterizing the thermal stability of ultraviolet Bragg gratings
}

\author{
Jacob Rathje ${ }^{\mathrm{a})}$ and Martin Kristensen \\ Research Center COM, Building 349, Technical University of Denmark, DK-2800 Lyngby, Denmark \\ Jens Engholm Pedersen \\ IONAS A/S, CAT Building, 347, DK-2800 Lyngby, Denmark
}

(Received 5 January 2000; accepted for publication 10 April 2000)

\begin{abstract}
We present a new method for determining the long-term stability of UV-induced fiber Bragg gratings. We use a continuous temperature ramp method in which systematic variation of the ramp speed probes both the short- and long-term stability. Results are obtained both for gratings written in $D_{2}$ loaded and nonloaded fibers. The results for the nonloaded fibers are in good agreement with those previously obtained. Precise predictions of the grating decay were made. We find good agreement with a broad trap energy distribution where the defects with the lowest energy decay first. For the $D_{2}$ loaded fiber grating we resolve two separate energy distributions, suggesting that two different defects are involved. The experiments show that complicated decays originating from various energy distributions can be analyzed with this continuous isochronal anneal method. The results have both practical applications in determining the long-term stability of fiber gratings and fundamental importance since they can be used to determine the energy distribution when using different fiber types and writing techniques. (C) 2000 American Institute of Physics.

[S0021-8979(00)03814-7]
\end{abstract}

\section{INTRODUCTION}

The widespread use of Bragg gratings in telecommunication and as sensor elements has created a need for an accurate determination of their stability. Both step isochronal $^{1-3}$ and isothermal anneals ${ }^{4-7}$ have been applied. Accelerated aging has been used to predict the long-term behavior at the designated operating temperature from the short-term behavior at elevated temperatures. By using step isochronal anneal on four gratings and varying the time interval for each grating the long-term stability has been derived. ${ }^{8}$

Erdogan et al. ${ }^{9}$ proposed an aging curve model based on an energy distribution of UV-induced defects. The gratings used were written in nonloaded fiber and the actual energy distribution was derived from a power law fit of the isothermal decay. The results have been reproduced for gratings written in different nonloaded fibers ${ }^{4-7}$ using somewhat different parameters. However, problems arise in obtaining satisfying fits for gratings written in hydrogen loaded fibers. ${ }^{4,5,10-12}$

We present here a novel continuous isochronal anneal method to obtain the aging curve. The procedure was inspired by Redhead. ${ }^{13}$ Redhead uses a continuous isochronal anneal with different heating rates to determine the energy and attempt frequency in the case of a single energy for thermal desorption of gases. We have expanded Redhead's method to cover the broad energy distribution of defects in germanium doped silica induced by UV light which is the basis for the aging curve model. We apply the continuous isochronal anneal to both $D_{2}$ loaded and nonloaded fibers.

${ }^{a)}$ Electronic mail: jr@com.dtu.dk

\section{THEORY}

For defects with energy, $E$, the decay rate for a first order reaction is determined by the Arrhenius relation,

$$
\frac{d \theta}{d t}=-\theta \nu e^{-E / k T},
$$

where $\theta$ is the population, $\nu$ is the attempt frequency, $k$ is the Boltzmann constant, $T$ is the temperature in kelvin, and $t$ is the time. During a continuous isochronal anneal the temperature is varied linearly as $T=T_{0}+\beta t$. With this the time decay rate in Eq. (1) can be rewritten to a temperature decay rate,

$$
\frac{d \theta}{d T}=-\theta \frac{\nu}{\beta} e^{-E / k T} .
$$

From this equation the energy, $E$, can be found from the temperature of the maximum decay rate when the attempt frequency, $\nu$, is known. Using two temperature sweeps with different heating rates, $\beta$, both energy, $E$, and the attempt frequency, $\nu$, can be determined. ${ }^{13}$

In the aging curve model ${ }^{9}$ the defects have an energy distribution rather than a single energy. The isothermal decay is determined from the energy distribution using a demarcation energy,

$$
E_{d}=k T \ln (\nu t) .
$$

When the energy distribution for UV-induced defects is sufficiently broad it is a good approximation to assume that the ratio of remaining defects with an energy, $E$, is a step function with the demarcation energy, $E_{d}$, separating the populated and unpopulated defects. 
When adjusting the continuous isochronal anneal to investigate an energy distribution we have to calculate the demarcation energy, $E_{d}(T)$, corresponding to the temperature, $T$. From Eq. (3) we see that annealing the grating at a temperature $T$ for a time $t$ is equivalent to annealing the grating at a temperature $T^{\prime}$ for a time $t^{\prime}$ when the following relation is satisfied:

$$
k T^{\prime} \ln \left(\nu t^{\prime}\right)=E_{d}=k T \ln (\nu t) .
$$

When this is rearranged we obtain the relation,

$$
t^{\prime}=\frac{1}{\nu}(\nu t)^{T / T^{\prime}} .
$$

The temperature sweep is divided into $N$ time intervals with length $\Delta t$ where the temperature in the $i$ th interval is considered constant $T_{i}$. When the $i$ th parameter set $\left(t_{i}, T_{i}\right.$, $\left.E_{d, i}\right)$ connected through Eq. (3) is known, the parameter set of the $(i+1)$ th interval is calculated by iteration using Eqs. (3) and (5),

$$
\begin{aligned}
& t_{i+1}=\Delta t+\frac{1}{\nu}\left(\nu t_{i}\right)^{T_{i} / T_{i+1}}, \\
& E_{d, i+1}=k T_{i+1} \ln \left(\nu t_{i+1}\right) .
\end{aligned}
$$

The initial parameter set is calculated by inserting into Eq. (3) the temperature, $T_{0}$, and the time, $t_{0}$, at which the grating has been kept after writing.

To obtain the correct attempt frequency, $\nu$, and energy distribution of the UV-induced defects, at least two grating decays with different heating rates are recorded. The two decays are then plotted as a function of the aging parameter, $E_{d}$, calculated for different trial attempt frequencies, $\nu_{t}$. Intuitively the decay curves will overlap when $\nu_{t}$ equals $\nu$. To illustrate this we have used Eq. (2) to calculate the temperature decay for a single energy defect with the energy $E=2.8 \mathrm{eV}$ and the attempt frequency $\nu=10^{13} \mathrm{~Hz}$. We have then used the procedure described above to transform the temperature scale into a demarcation energy scale. In Fig. 1 the decay rate is plotted for three different trial attempt frequencies, $\nu_{t}$. It can be seen that when the correct attempt frequency is used to calculate the demarcation energy the two curves overlap. Furthermore the demarcation energy at which the decay rate is maximum is the correct energy.

As shown in Fig. 1 the demarcation energies at which the decay rates are maximized are separated when $\nu_{t}$ is different from the correct attempt frequency. We would like to characterize the sensitivity of the continuous isochronal anneal to determine the attempt frequency, $\nu$. To do this we calculate the energy separation of the maximum decay rate between two different heating rates as a function of the error between $\nu_{t}$ and the correct attempt frequency, $\nu$. The result is shown in Fig. 2 where it should be noted that we have used logarithmic differences. As expected the larger the difference in heating rate the larger the energy separation between the two sweeps for the same error of trial attempt frequency. This means that the accuracy of the attempt frequency determination is increased when a larger difference in heating rate
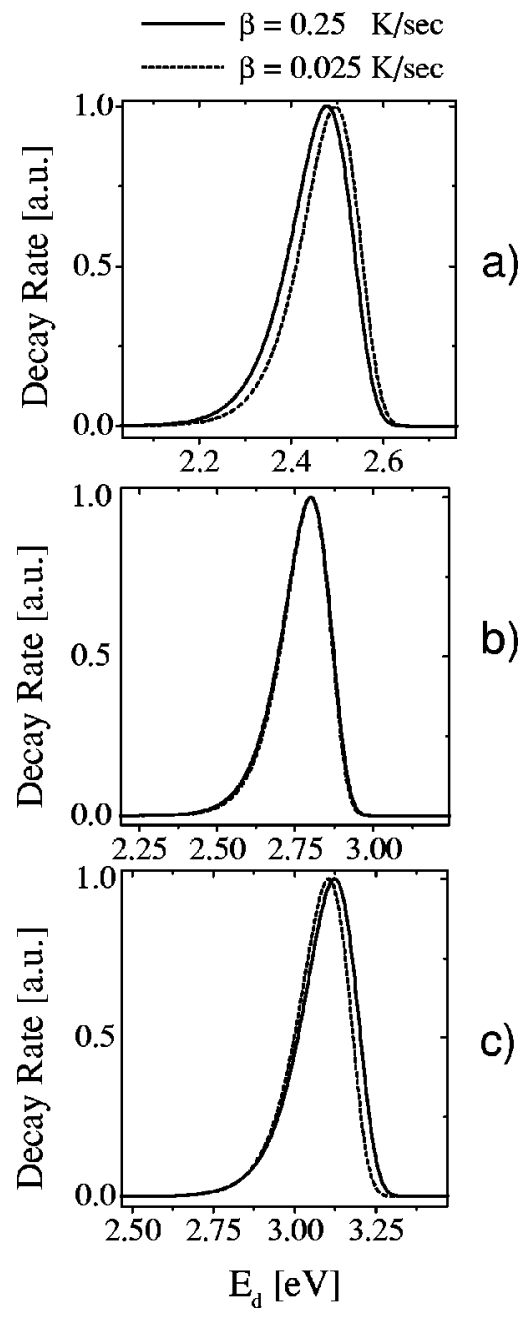

FIG. 1. Theoretical rate of decay for $2.8 \mathrm{eV}$ activation energy and an attempt frequency $\nu=10^{13} \mathrm{~s}^{-1}$. The decay rate at two values of $\beta$ is plotted against the demarcation energy, $E_{d}$, for different trial attempt frequencies, $\nu_{t}=$ (a) $10^{11}$, (b) $10^{13}$, and (c) $10^{15} \mathrm{~s}^{-1}$. It is seen that when the trial frequency is correct the curves overlap and are peaked at the correct energy.

is used. More precisely the accuracy of the attempt frequency is inversely proportional to the difference in the heating rates. Since the energy distribution is dependent on the attempt frequency used (see Fig. 1) this is also more accurately determined when a larger difference in the heating rates is used.

\section{EXPERIMENT}

The experimental setup is shown in Fig. 3. The oven can be programmed to heat up using a linear ramp with variable speed. During the experiment we record transmission spectra of the grating with an optical spectrum analyzer (OSA). We use this to monitor the temperature through the wavelength shift of the Bragg resonance, which we have calibrated from room temperature to $1125 \mathrm{~K}$. Further, the transmission spectra are used to calculate the grating strength $\kappa L$ determined from the transmission depth using the formula ${ }^{9}$

$$
\kappa L=\operatorname{arctanh}(\sqrt{R}),
$$




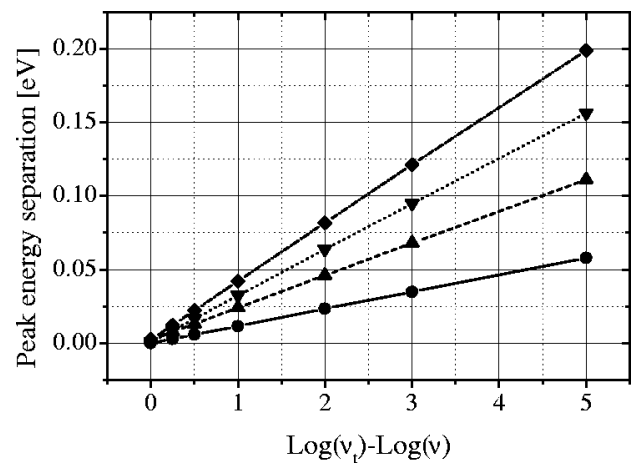

FIG. 2. Energy separation of the peak decay rate for different heating rates vs the error of trial attempt frequency. Note that the logarithmic difference is used.

where $R$ is the fraction of the light that is reflected, $L$ is the grating length, and $\kappa$ the coupling constant.

When annealing the grating it is important to avoid interference between core and cladding modes that have passed through the grating. This can, for example, be due to the use of a small piece of specialty fiber wherein the grating is UV written and that splicing the specialty fiber between the standard fiber allows for coupling of cladding and core modes at the splice intersections. The effect of interference is shown in Fig. 4 where on top of the regular decay oscillations are observed. To reduce this effect we use the same fiber all the way to the optical spectrum analyzer and make fiber loops to remove the cladding modes.

In the experiments a $15 \mathrm{~mol} \%$ germanium doped photosensitive fiber was used. The gratings are $5 \mathrm{~mm}$ long and approximately $6 \mathrm{~dB}$. We have inscribed gratings both in the loaded and unloaded fiber. In the unloaded fiber we used 600 pulses of $300 \mathrm{~mJ} / \mathrm{cm}^{2} /$ pulse from an excimer laser. In the loaded fiber we used 2300 pulses of $15 \mathrm{~mJ} / \mathrm{cm}^{2} /$ pulse.

\section{RESULTS}

During heating of a preannealed grating we observed that the depth of the grating changes with temperature. This phenomenon has recently also been reported by another group. ${ }^{8}$ We have plotted the change, $\kappa L$, as function of temperature in Fig. 5 and observe a linear relation. This linear relation gives a correction factor to $\kappa L$ as a function of the temperature. To correct for the error of $\kappa L$ calculated from the reflection is divided by the corresponding correction factor for that temperature. This correction has been done on all the results presented in this article.

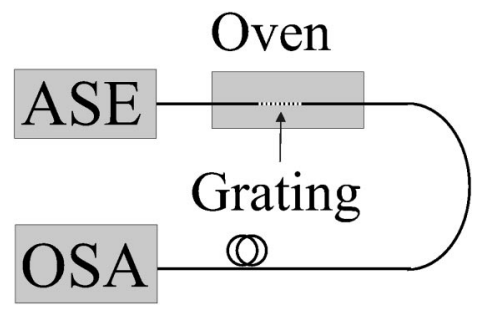

FIG. 3. Experimental setup.

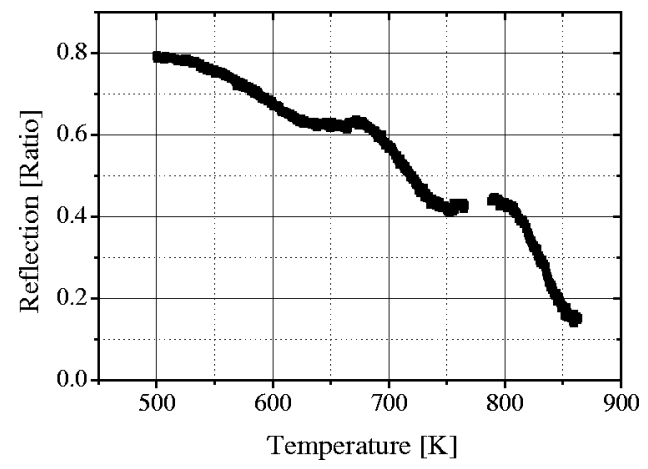

FIG. 4. Example of the effect of interference between core and cladding modes due to coupling of the core and cladding modes at the splice intersections on both sides of the grating.

The origin of the temperature induced change is unclear but we believe that it is due to a difference in the thermal index change between the core and the cladding. When the refractive index is increased more for the core than for the cladding, the mode is more confined to the core. The coupling efficiency is linearly proportional to the core overlap and therefore the reflection and $\kappa L$ increase.

\section{A. Annealing of gratings written in nonloaded fiber}

Three different temperature ramp speeds of $0.25,0.025$, and $0.0036 \mathrm{~K} / \mathrm{s}$ were used for the annealing of three similar gratings. The temperature decay of $\kappa L$ normalized to 1 has been plotted in Fig. 6. All three temperature decays have similar shape but are stretched along the temperature scale so that as expected the grating heated with the fastest heating rate survives to the highest temperature.

As described in Sec. II the temperature scale can be transformed to a demarcation energy scale when a trial attempt frequency, $\nu_{t}$, is assumed. We have calculated the decay of the normalized $\kappa L$ versus demarcation energy for $\nu_{t}$ in the interval from $10^{10}$ to $10^{18} \mathrm{~s}^{-1}$. To determine the correct attempt frequency, $\nu$, we calculate the square difference between the decay curves recorded with heating rates of 0.25 and $0.0036 \mathrm{~K} / \mathrm{s}$. These two are used since they have the largest difference in heating rates and therefore give the most accurate determination of $\nu$, as seen in Fig. 2. We have plotted the square difference values in Fig. 7 and it is seen that it has a minimum at $\nu_{t}=10^{13.5} \mathrm{~s}^{-1}$. This is in good agreement

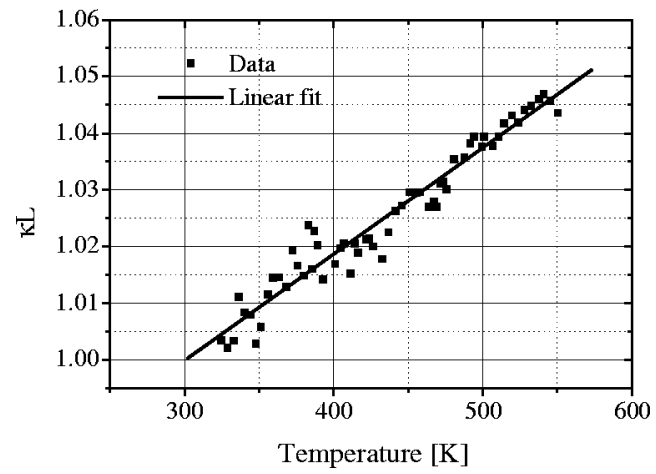

FIG. 5. When heating a preannealed grating a reversible change of $\kappa L$ is observed. As the fit shows the change is linear with temperature. 


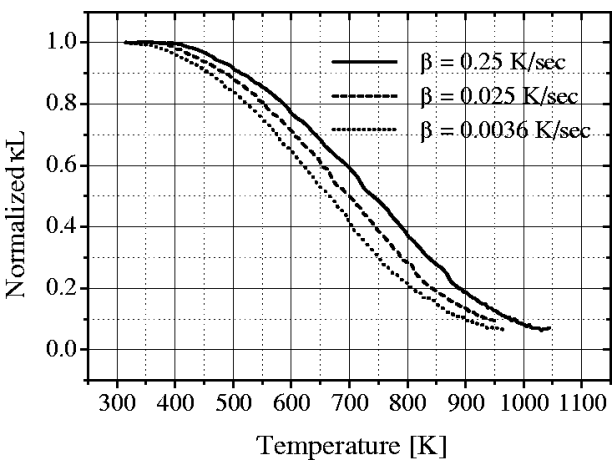

FIG. 6. Temperature decays of three similar gratings heated at different heating rates. It is seen that the shape of the decays is similar.

with the attempt frequency that we determined by qualitatively looking at the overlap of the decays versus demarcation energy when plotted for different trial attempt frequencies. The accuracy in the determination of the attempt frequency is better than an order of magnitude and we obtain the value $v=10^{13.5 \pm 1} \mathrm{~s}^{-1}$. The decays of the three gratings as function of demarcation energy, $E_{d}$, are shown in Fig. 8. It is seen that all three decays almost coincide.

Due to slight irregularities of the temperature ramp the decay of $\kappa L$ as function of $E_{d}$, Fig. 8, is smoother than as a function of temperature, Fig. 6 . When the demarcation energy is calculated using Eqs. (5) and (6) the time the grating is at a certain temperature is used and the irregularity therefore corrected. To obtain the final aging curve for the grating we calculate the average of the two decays with 0.25 and $0.0036 \mathrm{~K} / \mathrm{s}$ versus demarcation energy; see Fig. 9. These two decays are used since the minimum square differences in Fig. 7 were calculated from them. As can be seen the aging curve is very smooth even though it is obtained from the raw data.

As proposed by Erdogan et al. ${ }^{9}$ the aging curve for gratings written in nonloaded fibers can be described by

$$
\kappa L=\frac{A}{1+e^{E_{d}-E_{\text {center }} / \Delta E}},
$$

where the demarcation, $E_{d}$, is defined by Eq. (3), $E_{\text {center }}$ is the center energy, $\Delta E$ represents the width of the energy

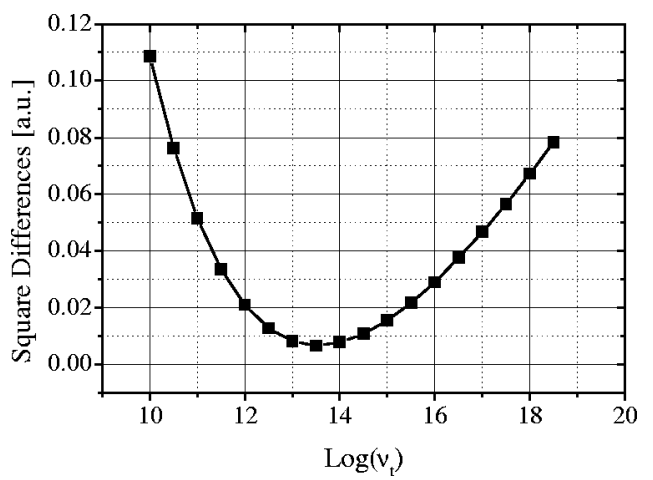

FIG. 7. Square difference values for the decays of 0.25 and $0.0036 \mathrm{~K} / \mathrm{s}$ plotted vs demarcation energy calculated for different trial attempt frequencies. A minimum is observed for $\log \left(\nu_{t}\right)=13.5$.

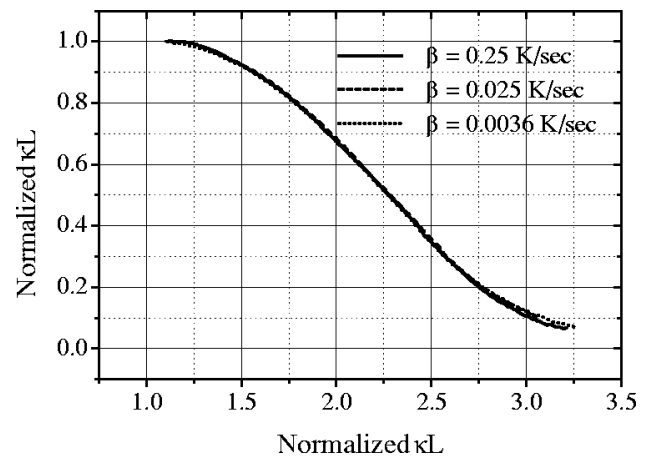

FIG. 8. Normalized $\kappa L$ for the three gratings heated at $0.25,0.025$, and $0.0025 \mathrm{~K} / \mathrm{s}$ vs demarcation energy, $E_{d}$, calculated for an attempt frequency $\nu=10^{13.5} \mathrm{~s}^{-1}$.

distribution, and $A$ is the initial value of $\kappa L$. We have fitted the data to Eq. (8) and as seen in Fig. 9 the fit shows that the data can be described well by Eq. (8). The fit gave the parameters $A=1.06, E_{\text {center }}=2.2 \mathrm{eV}$, and $\Delta=0.38 \mathrm{eV}$. In the fit we have allowed $A$ to have a value larger than the initial value of $\kappa L$ by assuming that this part of the grating has decayed prior to the anneal experiment. If $A$ was forced to be equal to the initial value of $\kappa L$ a small change of the parameters to $E_{\text {center }}=2.3 \mathrm{eV}$ and $\Delta=0.34 \mathrm{eV}$ was observed. The fit, however, was significantly worse than when $A$ is allowed to vary.

By differentiating the aging curve the underlying energy distribution of the defects is obtained. ${ }^{9}$ The energy distribution obtained from the raw data and the fit to Eq. (8) are shown in Fig. 10. The two energy distributions overlap very well around the center energy but the energy distribution from the raw data is cut off more sharply than the one from Eq. (8). At the low energy side this is due to the fact that the demarcation energy for the grating at the beginning of the anneal was nonzero and therefore the low energy defects were already erased. At the high energy side it is seen that the two distributions overlap within the error bars. The error bars increase as the grating strength decreases since $\kappa L$ is determined from the reflection through Eq. (7). The results in Figs. 9 and 10 are very encouraging for the continuous isochronal anneal since they show that the underlying energy

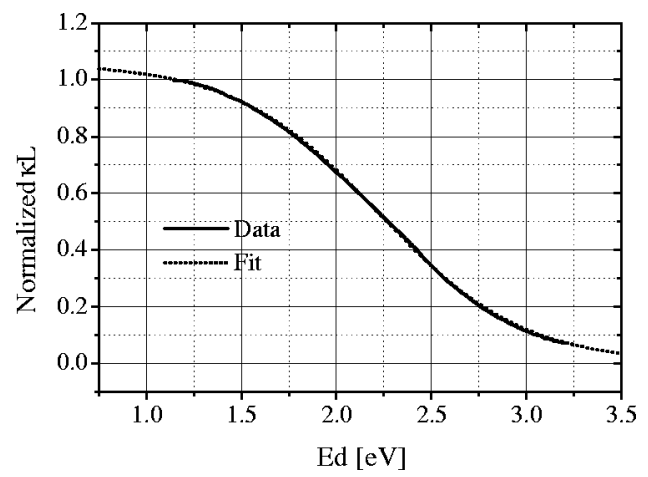

FIG. 9. Final aging curve found from the average of 0.25 and $0.0036 \mathrm{~K} / \mathrm{s}$. A fit of the aging curve to Eq. (8) gave the parameters $A=1.06$, $E_{\text {center }}=2.2 \mathrm{eV}$, and $\Delta=0.38 \mathrm{eV}$. The two curves overlap well. 


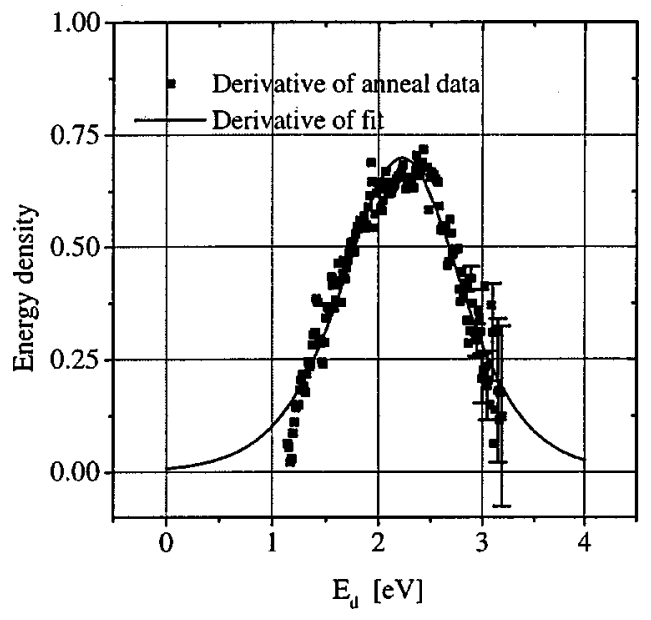

FIG. 10. Energy distribution of the defects obtained from differentiation of the aging curve in Fig. 9 and the corresponding fit to the power law. Good agreement is observed around the center energy.

distribution can be obtained without any assumptions. Further, the energy distribution of the defects that we found from the raw data is in good agreement with what was obtained by using the aging curve model.

To test the reliability of the aging curve obtained from the raw data we performed an experiment where a grating was heated with a ramp speed of $0.1 \mathrm{~K} / \mathrm{s}$ in two consecutive temperature sweeps. The first temperature ramp was from room temperature to $698 \mathrm{~K}$ where it was held for $2 \mathrm{~h}$. The second was from room temperature to $1023 \mathrm{~K}$ where the grating was erased. The actual and predicted decay are shown in Fig. 11 and it is seen that the two curves agree well.

The second temperature ramp is very interesting since no decay occurs at low temperature. After an intermediate temperature region the temperature in the second ramp follows the same decay as the first would have done if the temperature ramp had not been stopped at $698 \mathrm{~K}$. This directly shows that during the anneal the defects with low energy are erased first which was the physical picture proposed in the aging curve model. It also shows that using the temperature ramp isochronal anneal a cutoff energy distribution can be analyzed without problems. If the aging curve for a cutoff

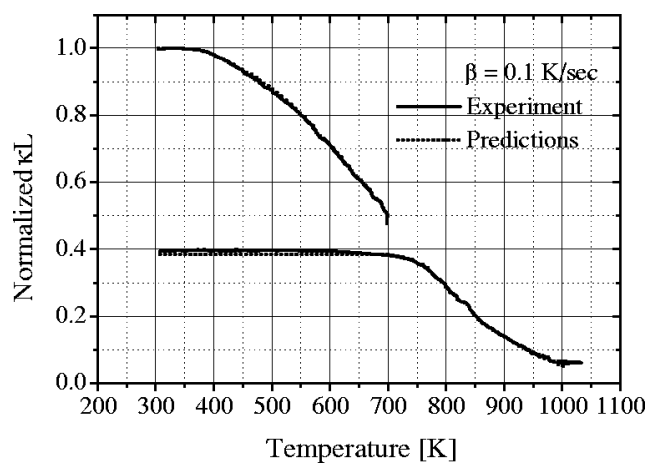

FIG. 11. Actual and predicted decay of two subsequent anneals of the same grating. The prediction based on the aging curve in Fig. 9 is in very good agreement with the experiment.

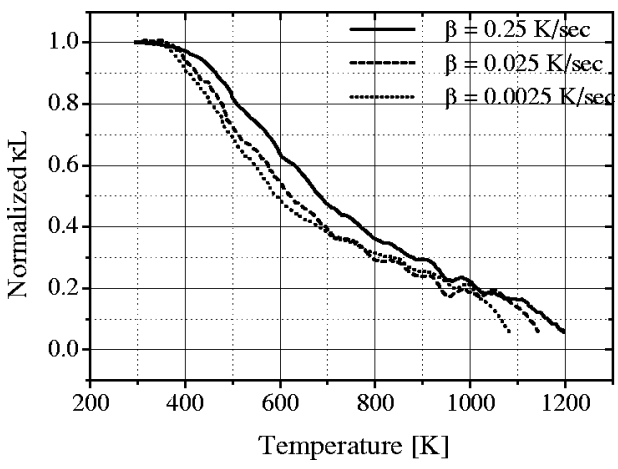

FIG. 12. Temperature decay of three similar gratings written in $D_{2}$ loaded fiber.

energy distribution were to be obtained by a fit to Eq. (8) the predicted decay would be erroneous. This problem has previously been discussed by Kannan et al. ${ }^{6}$

\section{B. Annealing of gratings written in $D_{2}$ loaded fiber}

In the second experiment the fiber was $D_{2}$ loaded before the gratings were inscribed. After the inscription the gratings were kept at $313 \mathrm{~K}$ for $90 \mathrm{~h}$ to allow the $D_{2}$ to diffuse out. Three similar gratings were heated at rates of $0.25,0.025$, and $0.0025 \mathrm{~K} / \mathrm{s}$, respectively, and the temperature decays are shown in Fig. 12. It is seen that at low temperature no decay occurs. This is due to the fact that the low energy defects have decayed before the experiment, similar to the behavior observed in Fig. 11 where the gratings were heated in two ramps.

To determine the correct attempt frequency we use the same least square difference method as that for the gratings written in the nonloaded fibers. The square difference values are shown in Fig. 13 and the minimal value is obtained for $\nu_{t}=10^{14.5} \mathrm{~s}^{-1}$. The decay of $\kappa L$ versus demarcation energy is shown in Fig. 14. It is seen that the decay is less smooth than that observed in Fig. 8 for the grating written in nonloaded fiber. We attribute the oscillations to interference between core and cladding modes, which was also observed earlier for gratings written in short pieces of fiber. We have tried to eliminate the oscillations but have only succeeded in

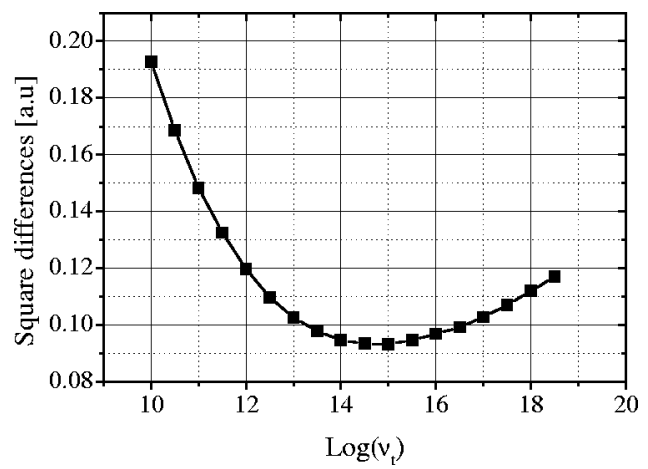

FIG. 13. Square difference values of the gratings written in $D_{2}$ loaded fiber and annealed at 0.25 and $0.0025 \mathrm{~K} / \mathrm{s}$. 


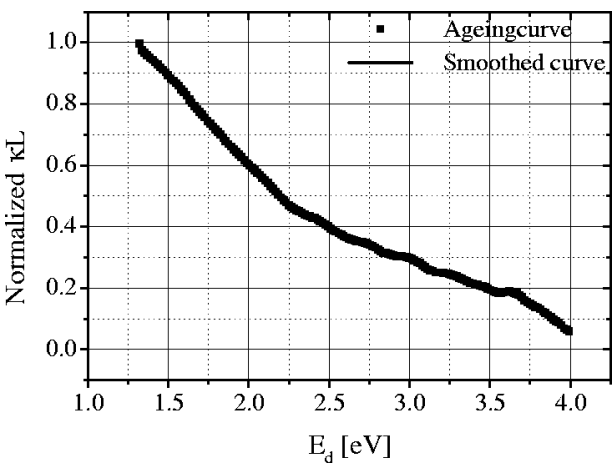

FIG. 14. Decay of grating written in $D_{2}$ - loaded fiber vs $E_{d}$ for the attempt frequency $\nu=10^{14.5} \mathrm{~s}^{-1}$.

reducing them. The accuracy in the determination of the attempt frequency is not as good as in the nonloaded case and we obtain the value $v=10^{14.5 \pm 2} \mathrm{~s}^{-1}$.

To get the aging curve the average of the decays at 0.25 and $0.0025 \mathrm{~K} / \mathrm{s}$ is used. This is shown in Fig. 15. Here it is seen that two different decay patterns are involved: when $E_{d}$ is below and above $3.6 \mathrm{eV}$. This suggests that two types of defects are involved. We have made the curve smoothing, which is also shown in Fig. 15. In Fig. 16 we have plotted the energy distribution obtained from differentiating the smoothed curve. It is seen that as expected there are two separate energy distributions. The above results show that a continuous isochronal anneal can be applied to different and complex energy distributions of the defects without any prior considerations of the energy distributions of the defects.

\section{CONCLUSION}

We have presented a continuous isochronal anneal method for analyzing the thermal stability of UV-induced defects. The continuous isochronal anneal technique is directly applicable with no assumptions regarding attempt frequency and energy distribution required to obtain the aging curve. We use a quantitative procedure based on square dif-

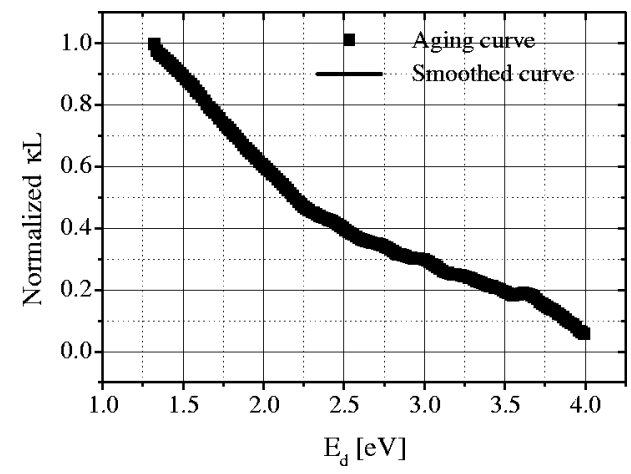

FIG. 15. Aging curve obtained from an average of the 0.25 and $0.0025 \mathrm{~K} / \mathrm{s}$ decays when the demarcation energy is calculated for frequency $\nu$ $=10^{14.5} \mathrm{~s}^{-1}$. The curve is not as smooth as that for the grating written in the unloaded fibered. We believe that this is due to interference but do not know the origin.

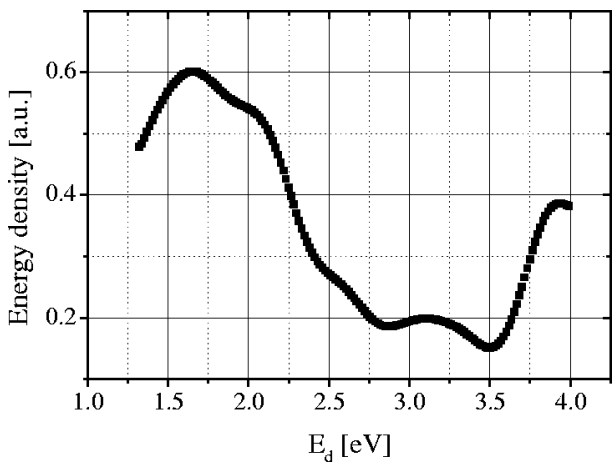

FIG. 16. Energy distribution of defects for gratings written in $D_{2}$ loaded fiber obtained by differentiating the smoothed curve. It can be seen that there are two different distributions, indicating two defect types.

ferences to determine the correct attempt frequency. This means that gratings written in any fiber using any writing condition can be analyzed. When the aging curve is established the underlying energy distribution can be obtained by differentiation.

The method was applied to gratings written in both $D_{2}$ loaded and nonloaded fiber. For the grating in nonloaded fiber the resulting aging curve is in agreement with what has previously been observed. Using the aging curve very accurate predictions of the temperature decay was made. For the gratings written in $D_{2}$ loaded fiber we observed two different energy distributions, indicating that two different defects were involved.

In conclusion we have shown that by applying the continuous isochronal anneal method we can obtain important information about the thermal stability and energy distribution of UV-induced defects.

\section{ACKNOWLEDGMENTS}

The authors would like to thank Erik V. Thomsen and Ole Hansen for fruitful discussions.

${ }^{1}$ J. Albert, B. Malo, K. O. Hill, F. Bilodeau, D. C. Johnson, and S. Thériault, Appl. Phys. Lett. 67, 3529 (1995).

${ }^{2}$ G. Meltz and W. W. Morey, Proc. SPIE 1516, 185 (1991)

${ }^{3}$ T. Taunay et al., J. Phys. D: Appl. Phys. 30, 40 (1997).

${ }^{4}$ H. Patrick, S. L. Gilbert, A. Lidgard, and M. D. Gallagher, J. Appl. Phys. 75, 2940 (1995)

${ }^{5}$ S. R. Baker, H. N. Rourke, V. Baker, and D. Goodchild, J. Lightwave Technol. 15, 1470 (1997).

${ }^{6}$ S. Kannan, J. Z. Y. Gou, and P. J. Lemaire, J. Lightwave Technol. 15, 1478 (1997).

${ }^{7}$ L. Dong and W. F. Liu, Appl. Opt. 36, 8222 (1997).

${ }^{8}$ D. Razafimahatratra, P. Niay, M. Dauay, B. Poumellec, and I. Riant, OSA Tech. Dig. Vol. 2, 216 (1999).

${ }^{9}$ T. Erdogan, V. Mizhari, P. J. Lemaire, and D. Monroe, J. Appl. Phys. 76, 73 (1994).

${ }^{10}$ R. J. Egan, H. G. Inglis, P. Hill, P. A. Krug, and F. Ouellette, OFC'96 Tech. Dig. Vol. 2, 83 (1996).

${ }^{11}$ G. Robert and I. Riant, OFC'97 Tech. Dig. Vol. 6, 180 (1997).

${ }^{12}$ I. Riant and B. Poumellec, Electron. Lett. 34, 1603 (1998).

${ }^{13}$ P. A. Redhead, Vacuum 12, 203 (1962). 